\title{
Definition of an envelope and life-of-mine production schedule for block caving mines under grade and material flow uncertainty
}

\author{
R Noriega University of Alberta, Canada \\ Y Pourrahimian University of Alberta, Canada
}

\begin{abstract}
One of the main concerns when determining an economic envelope and life-of-mine schedule for block caving projects is the potential dilution of the estimated grade model. Material flow and mixing, which coupled with the uncertainty associated with the grade estimation process can generate unreliable mine plans and unrealistic economic valuations at early stages of the project. This paper presents a stochastic mathematical programming model to incorporate 3D material flow and mixing, through the concept of a cone of movement, and grade uncertainty, through sequential Gaussian simulation (SGS), into the definition of an envelope and life-of-mine production schedule for block caving mines. The model maximizes the NPV of the project under operational constraints and can be used on the resource model at early stages of the project. The result is a more robust mine plan that aims to meet production and grade quality targets over the different scenarios and a more realistic NPV estimation for the project.
\end{abstract}

\section{Introduction}

Traditional mine plans are built using a single estimated deposit model, in which the mineral deposit is discretized into blocks. Each block has assigned attributes such as grade, rock type, and density, estimated from samples obtained from drill holes. Uncertainty in the estimation of metal grades and rock type modeling that arises due to the low volume of samples obtained during exploration programs have a high impact in the reliability of the projected cash flows and mining rates, leading to unexpected shortages and poor economic performance of the project. Adopting a risk-oriented approach, in which multiple simulated orebodies are built to capture the whole range of scenarios based on the available data, and building LOM plans that make use of them provide more robust schedules that take advantage and quantify geologic uncertainty (Dimitrakopoulos 2011).

Moreover, block caving operations rely on the gravitational flow of broken rock as the ore is extracted through the draw points at a specific elevation. This flow mechanism is related to the geotechnical and geological properties of the rock mass as well as the primary fragmentation or breakage method, leading to the mixing of the original estimated block model grades (Figure 1), which also affects the reliability of the mine plan (Laubscher et al. 2017).

The explicit incorporation of geological uncertainty in block caving planning has been very limited. (Dirkx et al. 2018) presented a comparative study of the scheduling of a particular caving footprint under geological and operational (hang-ups) uncertainties. The production schedules were obtained through a stochastic mathematical programming model. The results provided suggest that the "conventional" schedule, obtained with the kriging estimated deposit, is rendered unfeasible when subjected to the grade and hang-up scenarios. The incorporation of grade and hang-up uncertainty provided a more realistic and feasible estimate of the project economics. 


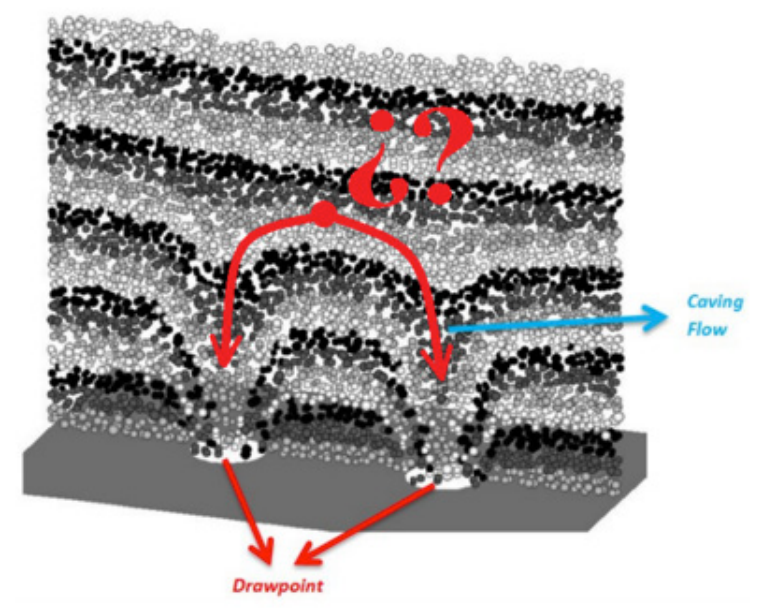

Figure 1 Schematic representation of material flow uncertainty in block caving (after Khodayari 2019)

(Khodayari \& Pourrahimian 2019) introduced an approach to incorporate material mixing explicitly within the mine planning workflow for block caving. Rather than modelling a detail flow mechanism, it is treated as a source of uncertainty reasoning that at this stage little is known about the response of the actual response of the rock mass, but multiple representative scenarios can be generated, and an optimization approach over the expected value of this set of potential scenarios can increase the value of the project as well as providing a more reliable mine plan. The concept of a cone of movement is developed (CoM), based on (Alvial 1992) observation on full-scale tests at El Teniente block caving mine, also mentioned in (Laubscher 2000). Potential movement of particles is modelled based on these observations as a cone of movement defined by a horizontal displacement (HD) and vertical slip angle (VSA). Multiple scenarios where tonnage and grade of the initial draw cone and slice model are modified based on this potential flow are generated to optimize the expected value of the project.

This paper presents a framework to integrate geological and material flow uncertainty into the block caving planning process, through the use of a stochastic programming optimization model taking as input a set of rock type and grade scenarios of an orebody with subsequent material flow simulations based on the CoM concept.

\section{Methodology}

The overall steps in the optimization framework are presented in Figure 2. An initial step aggregates the individual blocks into MUs, representing the column-wise extraction scheme of block caving, based on a desired draw point spacing and planned dilution. The aggregation maximizes the metal content of the units, calculated in reference to an undercut elevation, in order to cover the deposit while not imposing a boundary within the orebody and leaving this decision to the later production scheduling step.

Geological and material flow uncertainty are characterized by generating multiple scenarios. The scheduling algorithm becomes a stochastic optimization model which is transformed into its equivalent deterministic MILP formulation by the introduction of deviations variables. These deviation variables are assigned a cost that gives the user control over the risk of over- or under-production from defined targets in both ore tonnage and grade quality over the LOM plan. The objective function is formulated to maximize the expected value of the economic profit from the extraction of a unit while minimizing the total cost incurred due to deviations from targets over the multiple geological and flow scenarios. The framework comprises: 
1. Generate a set of block model realizations of the grade and rock type of a mineral deposit using SGS techniques.

2. Aggregate the individual blocks into production and mining units to reduce the number of variables to schedule while being representative of the block caving extraction scheme. The aggregation maximizes the expected metal content of the layout considering the multiple geological realizations.

3. From the set of geological realizations, generate material flow simulations using the CoM concept based on HD and VSA parameters to represent the geo-mechanical characteristics of the broken rock mass (Khodayari \& Pourrahimian 2019).

4. A Stochastic Integer Programming (SIP) model is formulated to generate a production schedule and mining envelope that maximizes the expected NPV of the project while minimizing the deviations in production targets due to the geological and material flow uncertainty.

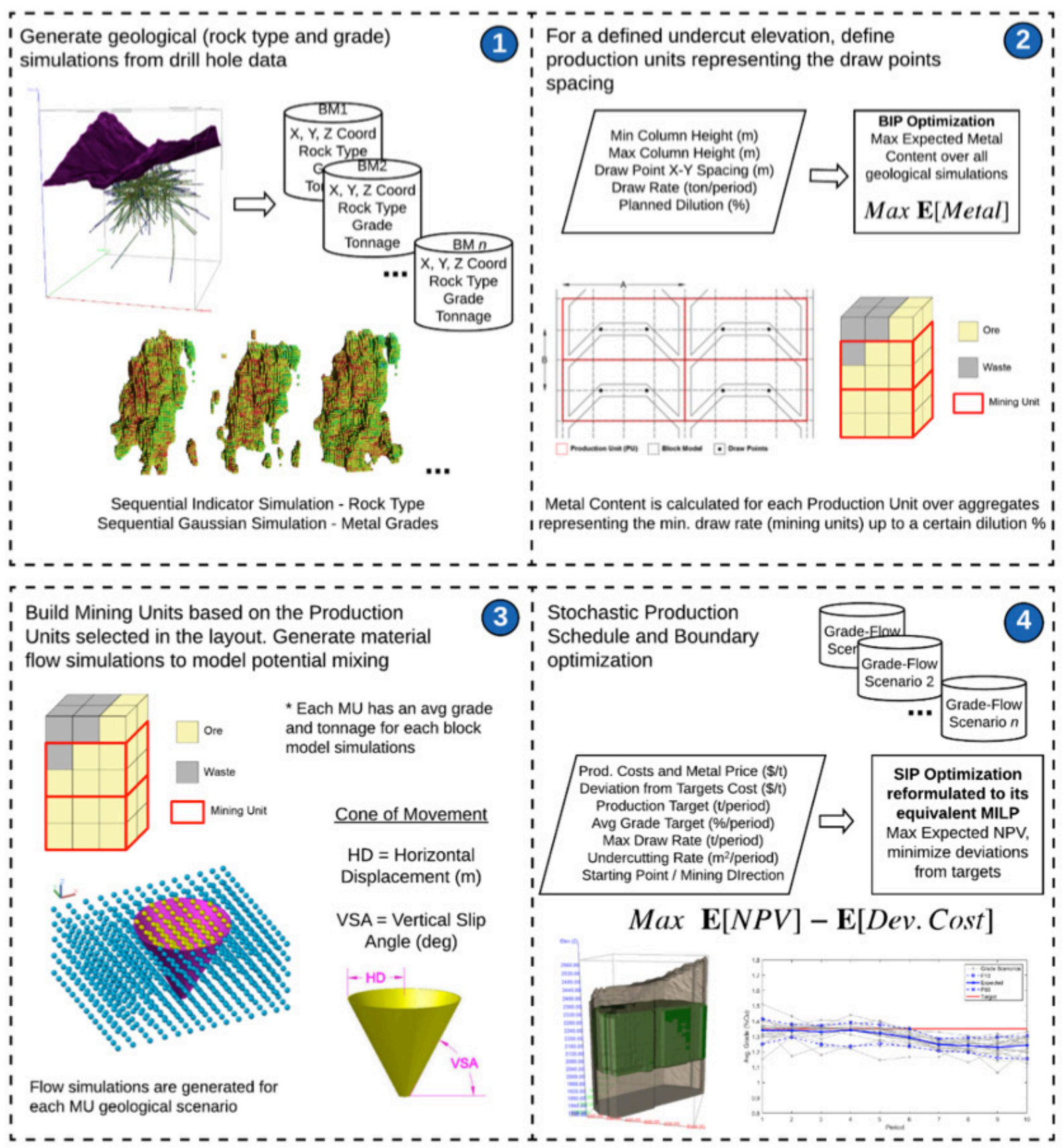

Figure 2 Summary of the optimization framework for the integration of geological and material flow uncertainty in the block caving mine planning process 


\subsection{Mining unit aggregation}

In this stage, the blocks are aggregated into mining units (MU), with the objective of maximizing the expected metal content contained within, for scheduling purposes. Due to the operating conditions of block caving mines, the MU aggregation is on a column-wise scheme based on a reference undercut elevation from which the overlying material comprise the mineral reserves. To simplify this procedure, the columns are represented as production unit (PU) in a plan view at the undercut section, based on draw point spacing. These PUs span the columns up to a certain height, which at this stage is determined either by reaching a predefined maximum height, or once a dilution percentage limit is surpassed to account for the deposit ore-waste boundaries. Dilution is evaluated based on the potential MU from each PU, as the percentage fraction of waste tonnage in relation to the total tonnage of the unit.

The aggregation step has an impact in the resulting optimal life-of-mine plan as information is lost when grouping resource blocks into larger extraction units. However, aggregation techniques have been widely proposed and used to reduce the computing time of optimization models regarding mine planning problems as in (Osanloo et al. 2008; Tabesh \& Askari-Nasab 2019), with some particular applications to block caving in (Weintraub et al. 2008; Pourrahimian 2013; Nezhadshahmohammad et al. 2018; Nezhadshahmohammad \& Pourrahimian 2018). Quantification of the impact of the aggregation step in the optimal solution is outside the scope of the work presented here.

A schematic of the block aggregation terminology and representative units is presented in Figure 3. The following assumptions are made for the PU layout aggregation:

- The aggregation is done to the highest integer of the desired draw point spacing in relation to the block size.

- Each PU contains multiple MU that span vertically up to a certain maximum height or maximum dilution \%. The metal content and dilution \% evaluation is carried out on MU aggregates at a time for each column.

- No mixing or dilution is considered at this stage.

- The expected metal content is calculated as the average metal content amongst all realizations, this is used for the coefficient calculation of the stochastic model. For kriging mine plans, the kriging metal content is used.
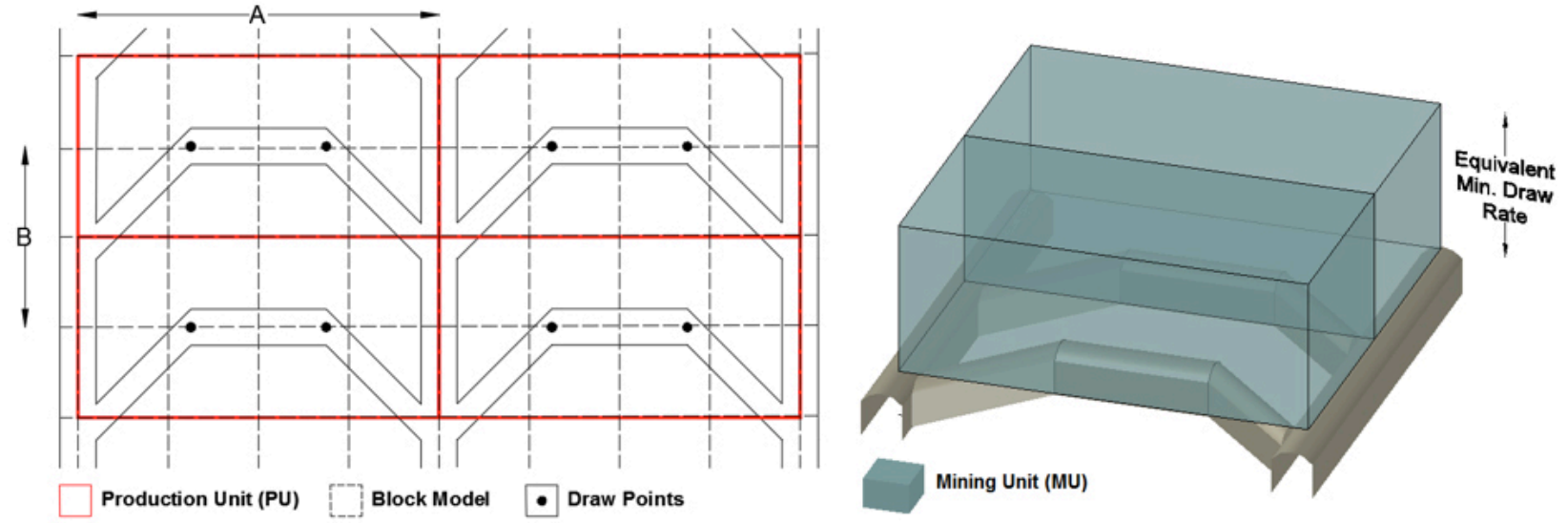

Figure 3 Aggregation of blocks into Mining Units for scheduling

\subsection{Material flow scenarios}

For each PU, the cone of movement is initially placed at the bottom of the lowest MU after a desired point of entry dilution, the point of entry dilution defines the height at which draw interaction between 
adjacent draw points occurs. For each MU, a set of candidate blocks is defined as those blocks whose center points lie within the cone (Figure 4).

From this candidate blocks, random blocks are drawn forming a sample set. This sample set keeps drawing random blocks from the candidate set until the total tonnage contained matches the tonnage of that particular MU (considering a small tolerance to account for density differences between ore and waste). This means that the MU has been "filled" with blocks from the resource model that can potentially show up when it is extracted. The procedure is repeated for every MU, flagging "used" blocks to avoid having the same individual block fall in MU that have overlapping cones (adjacent or overlying ones).

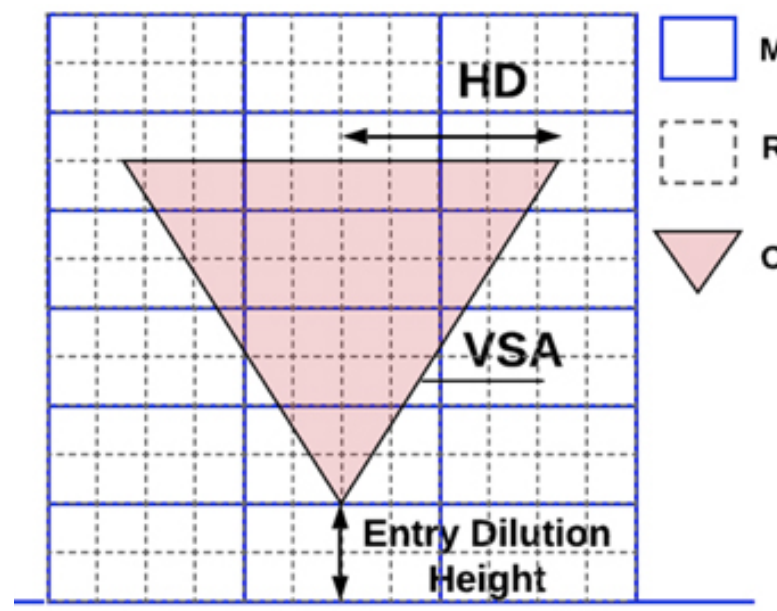

Mining Units

Resource Blocks

Cone of Movement

Figure 4 Schematic of the CoM and potential blocks sampled to update each MU grade and tonnage in each scenario

\subsection{LOM production scheduling incorporation geological and material flow uncertainty}

Each MU has a set of possible tonnages and grades for each geology-material flow simulation performed, that represent the uncertainty based on the estimation procedure and behavior of block caving mines. The following section details the formulation of an optimization model that takes as an input the whole set of grade-tonnage scenarios, rather than a single estimate, and generates a mine plan that maximizes the NPV of the project while minimizing the potential deviations due to the mentioned uncertainties. The following assumptions are made in the production scheduling optimization model.

- The decision to extract a MU is binary, which means that for each period it will decide whether to extract the whole unit or not.

- No hard-fixed cut-off grade is considered. The optimization model decides whether to initiate extraction within a PU and up to which height based on its overall discounted expected profit contribution to the mine plan NPV.

- To estimate expected values in the objective function coefficients, arithmetic average is used due to the assumption that the geological simulations are equiprobable realizations.

- Uncertainty is assumed to impact production targets and grade quality per period, and the model is developed to minimize deviations in these outputs only.

Table 1 to Table 4 show the indices, sets, decision variables and parameters used in the formulation of the SIP model. 
Table 1 Indices used in the model

\begin{tabular}{|c|c|}
\hline Indices & \\
\hline$u \in\{1, \ldots, U\}$ & Index for all mining units \\
\hline$t \in\{1, \ldots, T\}$ & Index for all periods \\
\hline$c \in\{1, \ldots, C\}$ & Index for all production units \\
\hline$s \in\{1, \ldots, S\}$ & Index for all geological and material flow realizations \\
\hline$u_{c}^{1}$ & Index for the first or lowest mining unit within the production unit c \\
\hline
\end{tabular}

\section{Table 2 Sets used in the model}

\begin{tabular}{|c|c|}
\hline Sets & \\
\hline$C^{c}$ & $\begin{array}{l}\text { Set containing the mining units that are within the production unit c. Each set has } \\
\qquad \text { a total number of elements of } N(C)\end{array}$ \\
\hline$V^{u, c}$ & $\begin{array}{l}\text { Single element set containing the mining unit directly below unit } u \text { of the } \\
\text { production unit c. Used for vertical precedence constraints }\end{array}$ \\
\hline$H^{u, c}$ & $\begin{array}{l}\text { Set containing the production units that have to be opened before the extraction } \\
\text { of the mining unit } u \text { of the production unit } c \text {, based on the mining direction. Each } \\
\text { set has a total number of elements of } N\left(H^{u, c}\right)\end{array}$ \\
\hline$A^{c}$ & $\begin{array}{l}\text { Set containing all adjacent production units to the unit c for cave back slope } \\
\text { constraint. Each set has a total number of elements of } N\left(A^{c}\right) N\left(A^{c}\right)\end{array}$ \\
\hline
\end{tabular}

Table 3 Decision variables used in the model

\begin{tabular}{|l|r|}
\hline \multicolumn{1}{|c|}{ Decision Variables } & Binary variable controlling the decision to extract the mining unit u of \\
production unit c in period t
\end{tabular}


Table 4 Parameters used in the model

\begin{tabular}{|c|c|}
\hline Parameters & \\
\hline MetalPrice & Metal price per ton of metal $(\$ / t)$ \\
\hline$p^{+}$ & Cost for positive deviation (overproduction) of ore tonnage $(\$ / t)$ \\
\hline$p^{\square}$ & Cost for negative deviation (shortage) of ore tonnage $(\$ / t)$ \\
\hline$q^{+}$ & Cost for positive deviation (over upper bound) of grade quality range $(\$ / t)$ \\
\hline$q^{\square}$ & Cost for negative deviation (under lower bound) of grade quality range ( $\$ / t)$ \\
\hline $\operatorname{Rec}$ & Metallurgical recovery of the processing operations (\%) \\
\hline$g_{u}$ & Grade of the mining unit $\mathrm{u}$ of production unit $\mathrm{c}(\mathrm{g} / \mathrm{t})$ \\
\hline Ton $_{u}$ & Tonnage of mining unit $u$ of production unit $\mathrm{c}(\mathrm{t})$ \\
\hline$S_{u_{c}^{1}}$ & $\begin{array}{l}\text { Planar surface area of production unit c }\left(\mathrm{m}^{2}\right) \text {, based on the first (lowest) MU of } \\
\text { the PU }\end{array}$ \\
\hline Height $_{u}$ & Height of mining unit $u(m)$ \\
\hline$M C$ & Mining cost per ton of ore in period $t(\$ / t)$ \\
\hline$P C$ & Processing cost per ton of ore in period $t(\$ / t)$ \\
\hline OpCost $c_{c}^{t}$ & $\begin{array}{l}\text { Development cost of production unit } c \text {, accounted for when extracting the } \\
\text { first (lowest) } \mathrm{MU}, u_{c} \text {, of production unit c }(\$)\end{array}$ \\
\hline$i$ & Discount rate (\%) \\
\hline $\bar{M}^{t}$ & Ore production target in period $\mathrm{t}(\mathrm{t})$ \\
\hline $\bar{G}^{t}$ & Average grade upper bound on period $t$ \\
\hline$\underline{G}^{t}$ & Average grade lower bound on period $t$ \\
\hline$\underline{D R}, \overline{D R}$ & Minimum and maximum Draw Rate of production units per period (t/period) \\
\hline MaxOpRate ${ }^{t}$ & Maximum undercutting rate $\left(\mathrm{m}^{2} /\right.$ period) \\
\hline MaxAbsDiffc $c_{c}^{c^{\prime}}$ & $\begin{array}{l}\text { Maximum allowable absolute difference in height between production unit } \\
\mathrm{c} \text { and each member c' of its adjacent set Ac to maintain cave back slope } \\
\text { constraints }\end{array}$ \\
\hline
\end{tabular}

The objective function is presented in Equation 1. The first term is the expected NPV from the extraction of the $\mathrm{MU}$ in a particular period, the second term comprises the opening or development cost for each $\mathrm{PU}$, and the third term accounts for the expected cost from the deviations in ore production targets and grade quality bounds amongst the realizations.

$$
\begin{aligned}
& \operatorname{Max} \sum_{t=1}^{T} \sum_{u=1}^{U} E\left[N P V_{u}^{t}\right] \cdot \mathrm{z}_{u}^{t}-\sum_{t=1}^{T} \sum_{c=1}^{C} \frac{\operatorname{OpCost}_{c}^{t} \cdot z_{1, c}^{t}}{(1+i)^{t}}- \\
& \frac{1}{S} \sum_{s=1}^{S} \sum_{t=1}^{T} \frac{\left(p^{+} \cdot \operatorname{tonDev}_{t, s}^{+}+p^{-} \cdot \operatorname{tonDev}_{t, s}^{-}+q^{+} \cdot \operatorname{gradeDev}_{t, s}^{+}+q^{-} \cdot \operatorname{gradeDev}_{t, s}^{-}\right)}{(1+g)^{t}}
\end{aligned}
$$

The following set of constraints are included in the formulation: 
- Mining capacity target (Equation 2)

This constraint controls the total mining capacity on a period basis. It ensures that the total tonnage of material extracted in each period is as close as possible to the desired target amongst all the realizations.

$$
\sum_{u=1}^{U} T_{u} \cdot z_{u}^{t}-\operatorname{tonDev}_{t, s}^{+}+\operatorname{tonDev}_{t, s}^{-}=\bar{M}^{t} \quad \forall t, s
$$

- Grade blending range (Equation 3 and Equation 4)

This constraint ensures that the average grade of the material extracted in each period is within the desired range, by the definition of lower and upper bounds, amongst all the realizations.

$$
\begin{aligned}
& \frac{\sum_{u=1}^{U} g_{u} \cdot \operatorname{Ton}_{u} \cdot z_{u}^{t}}{\sum_{u=1}^{U} \operatorname{Ton}_{u} \cdot z_{u}^{t}}-\operatorname{gradeDev}_{t, s}^{+} \leq \bar{G}^{t} \quad \forall t, s \\
& \frac{\sum_{u=1}^{U} g_{u} \cdot \operatorname{Ton}_{u} \cdot z_{u}^{t}}{\sum_{u=1}^{U} \operatorname{Ton}_{u} \cdot z_{u}^{t}}+\operatorname{gradeDev}_{t, s}^{-} \geq \underline{G}^{t} \quad \forall t, s
\end{aligned}
$$

- Maximum draw rate (Equation 5)

This constraint guarantees that the vertical extraction rate from each PU is less or equal than a maximum draw rate established.

$$
\sum_{c=1}^{N\left(C^{c}\right)} T_{u} \cdot z_{u}^{t} \leq \overline{D R} \quad \forall c, t
$$

- Undercut development rate (Equation 6)

This constraint controls the number of PU units opened on each period, reflecting the maximum undercut area that can be developed based on an established capacity.

$$
\sum_{c=1}^{C} S_{u_{c}^{1}} \cdot z_{u}^{t} \leq \text { MaxOpRate } \quad \forall t
$$

- Maximum adjacent height of draw (Equation 7)

This constraint ensures that the relative height of draw between adjacent columns is within a certain specified range, which is established as number of $\mathrm{MU}$ in order to be coherent with the aggregation level, to control the cave back slope during the mine life.

$$
\sum_{u=1}^{U}\left[\text { Height }_{u} \cdot z_{u}^{t}\right]-\sum_{u=1}^{U}\left[\text { Height }_{u} \cdot z_{u, c^{\prime}}^{t}\right] \leq \text { MaxAbsDiff }_{c}^{c^{\prime}} \quad \forall c, t \forall c^{\prime} \in A^{c}
$$

- Mining precedence (Equation 8 and Equation 9)

This constraint forces the sequence to be feasible based on the starting position and mining advancement direction. Two types of precedence are defined: horizontal precedence which control the opening of PU on the undercut layout, and vertical precedence which control the extraction of MU within each PU. 


$$
\begin{gathered}
z_{u}^{t} \leq z_{V^{u, c}}^{t}+z_{V^{u, c}}^{t-1} \quad \forall u, t \\
N\left(H^{u, c}\right) \cdot z_{u_{c}^{t}, c}^{t} \leq \sum_{t=1}^{T} \sum_{c=1}^{C} z_{u_{c}^{t}, c}^{t} \quad \forall c, \mathrm{t}
\end{gathered}
$$

- Reserves (Equation 10)

This constraint ensures that each $\mathrm{MU}$ is either extracted once or not extracted at all.

$$
\sum_{t=1}^{T} z_{u}^{t} \leq 1 \quad \forall u
$$

The model is implemented in a MATLAB environment, using IBM CPLEX optimization engine which uses a branch-and-cut method to obtain a solution under a given MILP gap. To improve the computing solution time, the earliest start pre-processing algorithm presented by (Topal 2008) is implemented. The precedence constraints of the mining units, both vertical and horizontal, together with the mining capacities, maximum draw rate and relative adjacent height of draw constraints, can be used in order to establish the earliest possible period that a mining unit can be extracted. Therefore, all variables related to the extraction of the MU at an earlier period can be eliminated from the problem.

A sliding time window heuristic (STWH), as adopted for mine planning by (Cullenbine et al. 2011), is also implemented in order to further reduce the computing times and make the optimization framework more useful to mine planners.

\section{Case study}

A copper deposit was used to test the application of the model discussed above, for simplicity two rock types were considered during the geostatistical simulation procedure representing ore and waste. A set of 20 orebody simulations were generated to run the optimization model and evaluate the results. The evaluation of the number of simulations required to adequately transfer the orebody uncertainty into the mine plan is outside of the scope of the work presented here, however a sensitivity analysis is suggested. Mining operational parameters were selected to be representative of an average block caving mine, while economic parameters were chosen to be representative of current conditions. The workflow follows the generation of rock type scenarios using a Sequential Indicator Simulation method and generating copper grade realization within the generated rock types using a Sequential Gaussian Simulation algorithm. A view of different orebody realizations showing the copper grade is presented in Figure 5.
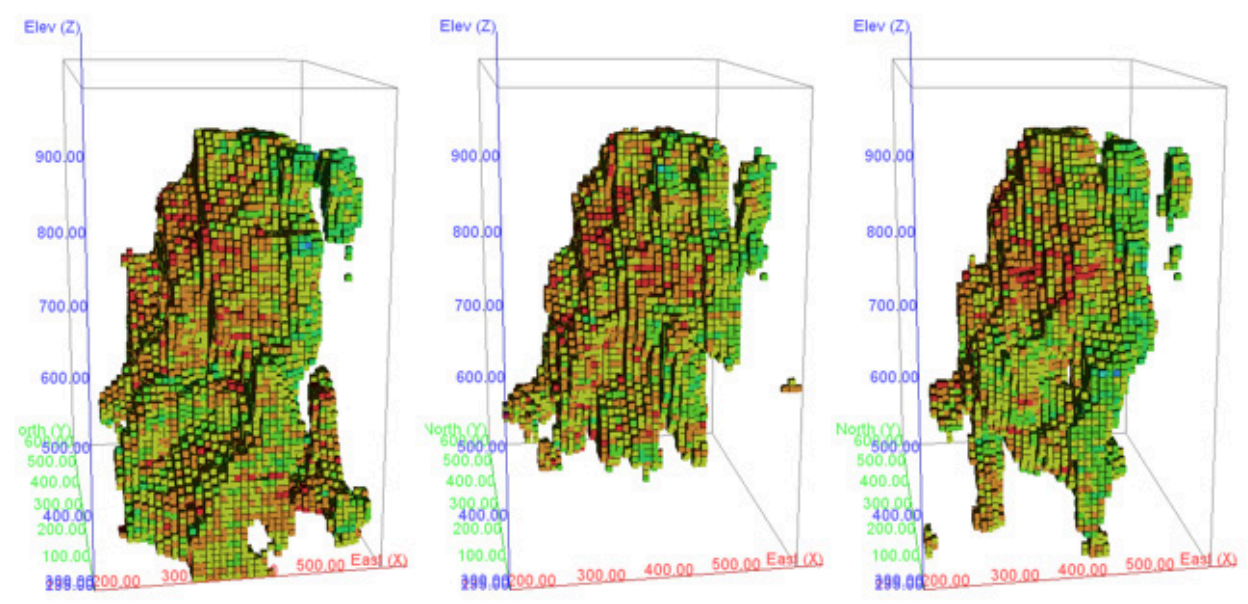

Figure 5 A set of realizations showing the different possible orebody scenarios based on the data available for modelling 
The economic parameters used for the valuation of the schedules during the optimization model are presented in Table 5. These parameters can be defined by the user to evaluate different economic scenarios.

Table 5 Economic parameters used for the testing of the model in the case study

\begin{tabular}{|c|c|c|}
\hline Description & Parameter & Value \\
\hline Selling Price $(\$ / t)$ & MetalPricet & 5,500 \\
\hline Mining Cost $(\$ / t)$ & MineCostt & 9.3 \\
\hline Processing Cost $(\$ / t)$ & ProcessCostt & 18.4 \\
\hline Recovery $(\%)$ & Rec & 88.7 \\
\hline Discount Rate $(\%)$ & $i$ & 12 \\
\hline $\begin{array}{c}\text { Development Cost } \\
(\$ / P U)\end{array}$ & OpCost $_{c}^{t}$ & 150,000 \\
\hline
\end{tabular}

The operational parameters are presented in Table 6. The PU dimensions represent an approximation of the draw point spacing, however it is constrained by the resource model block size. The minimum draw rate was set at $35 \mathrm{kton} /$ period, which along with the draw point spacing would be representative of a vertical extraction rate of about $20 \mathrm{~m} /$ period. The draw rate is considered to be the constant for the column throughout the mine life. This is also used to aggregate the blocks in the vertical direction into $\mathrm{MU}$, once the optimum PU layout has been obtained.

Table 6 Operational parameters used for the testing of the model in the case study

\begin{tabular}{|c|c|c|}
\hline Description & Parameter & Value \\
\hline PU Dimensions & & $30 \mathrm{~m} \times 20 \mathrm{~m}$ \\
\hline Maximum Column Height & & $300 \mathrm{~m}$ \\
\hline Minimum Column Height & & $60 \mathrm{~m}$ \\
\hline No. of Periods & $T$ & 10 \\
\hline Minimum Draw Rate & $\underline{D R}$ & $35(\mathrm{kton} /$ period $)(\sim 20 \mathrm{~m} /$ period $)$ \\
\hline Maximum Draw Rate & $\overline{D R}$ & $100(\mathrm{kton} /$ period $)(\sim 60 \mathrm{~m} /$ period $)$ \\
\hline Undercut Development Rate & MaxOpRate & $12,000\left(\mathrm{~m}^{2} /\right.$ period $)$ \\
\hline Maximum Relative Height of Draw & MaxAbsDiff $c_{c}^{c^{\prime}}$ & $70(\mathrm{kton} /$ period $)(\sim 2 \mathrm{MU})$ \\
\hline Mining Starting Point & & $380 \mathrm{mE}, 230 \mathrm{mN}$ \\
\hline Mining Direction Azimuth & & $335^{\circ}$ \\
\hline Convex Front Angle & & $170^{\circ}$ \\
\hline
\end{tabular}

The maximum relative height of draw between PUs is used to approximate the cave back slope as the extraction progresses to allow for safe extraction and efficient draw control. The definition should consider the dimensions of the PU and the approximate height of the MU based on the spacing and draw rates evaluated. For the case study, a draw difference of $70 \mathrm{kton} /$ period was considered, which represents two $\mathrm{MU}$ and a maximum cave back angle of around $60^{\circ}$.

The mining direction and starting point are used to build the precedence relations for the MU. The user has control to decide the starting point of the caving operation, the general caving direction defined by an azimuth and the angle of the convex face. 
The other parameters give the user flexibility to build a feasible mine plan that follows the caving extraction mechanisms. The unit of the periods are considered years, as the model is formulated for long-term planning.

The operating parameters for the schedule were determined by different trials to obtain a feasible schedule from the mineral resource. An ore production target is defined, which the scheduling model tries to match as close as possible, with deviations being penalized. A minimum and maximum average grade sent to mill during each period is set to guarantee a correct operation of the mill process. The production target was set at 3.5 with ramp-up and ramp-down periods and the minimum average grade sent to mill was set at $1.2 \% \mathrm{Cu}$ with the maximum at $1.5 \% \mathrm{Cu}$.

Finally, the deviation cost parameters are defined, which control the risk profile and how the scheduling algorithm manages the multiple orebody geological and flow simulations (Table 7). A simple concept is to consider that the cost of underproduction of ore tonnage represents the lost revenue from that shortfall. Therefore, the cost of deviation from ore production targets is related to the average revenue obtained from the extraction of a ton of ore. The deposit average grade can serve to provide an initial estimate. Higher values would lead to the optimization model to prioritize avoiding shortfalls in ore production amongst all simulations rather than obtaining the highest overall NPV. The similar principle applies for the overproduction of ore tonnages. Overproduction cost can also be linked to a differential cost that can be potentially incurred in order to manage the excess production.

\section{Table 7 Deviation costs and geological discount rate assigned for the testing of the model in the case study}

\begin{tabular}{|c|c|c|}
\hline Description & Parameter & Value \\
\hline Cost for positive deviation (overproduction) of ore tonnage $(\$ / t)$ & $P^{+}$ & 60 \\
\hline Cost for negative deviation (shortage) of ore tonnage $(\$ / t)$ & $P^{-}$ & 120 \\
\hline $\begin{array}{l}\text { Cost for positive deviation (over upper bound) of grade quality } \\
\text { range }(\$ / t)\end{array}$ & $q^{+}$ & 6,000 \\
\hline $\begin{array}{l}\text { Cost for negative deviation (under lower bound) of grade quality } \\
\text { range }(\$ / t)\end{array}$ & $q^{-}$ & 12,000 \\
\hline Geological discount rate (\%) & $g$ & 15 \\
\hline
\end{tabular}

The estimation of the cost for positive and negative deviations of average grade sent to mill follows a similar principle. The cost of shortfall or positive deviation of metal is related to the revenue obtained from the selling of one ton of it. The selling cost of the metal of interest could serve as a reference for an initial estimation for the deviation cost of average grades sent to the mill.

The geological discount rate is the final parameter related to the uncertainty based production scheduling model. This rate serves as a discount factor for the cost associated with the deviations due to the variation between the simulated models. High values would prioritize minimizing deviations on the initial periods at the cost of potentially lower NPV, while lower values would lead to higher deviations allowed in the initial periods.

The stochastic formulation was used through different elevations of the set of orebody realizations in order to define the undercut level with the highest expected NPV and show its mine plan and project KPIs. Figure 6 shows the stochastic caving envelope at the optimal undercut elevation. For displaying and comparison purposes, the grades shown are the expected copper grade from all the geology realizations with a deterministic orebody model in the background generated through traditional kriging estimation techniques. 


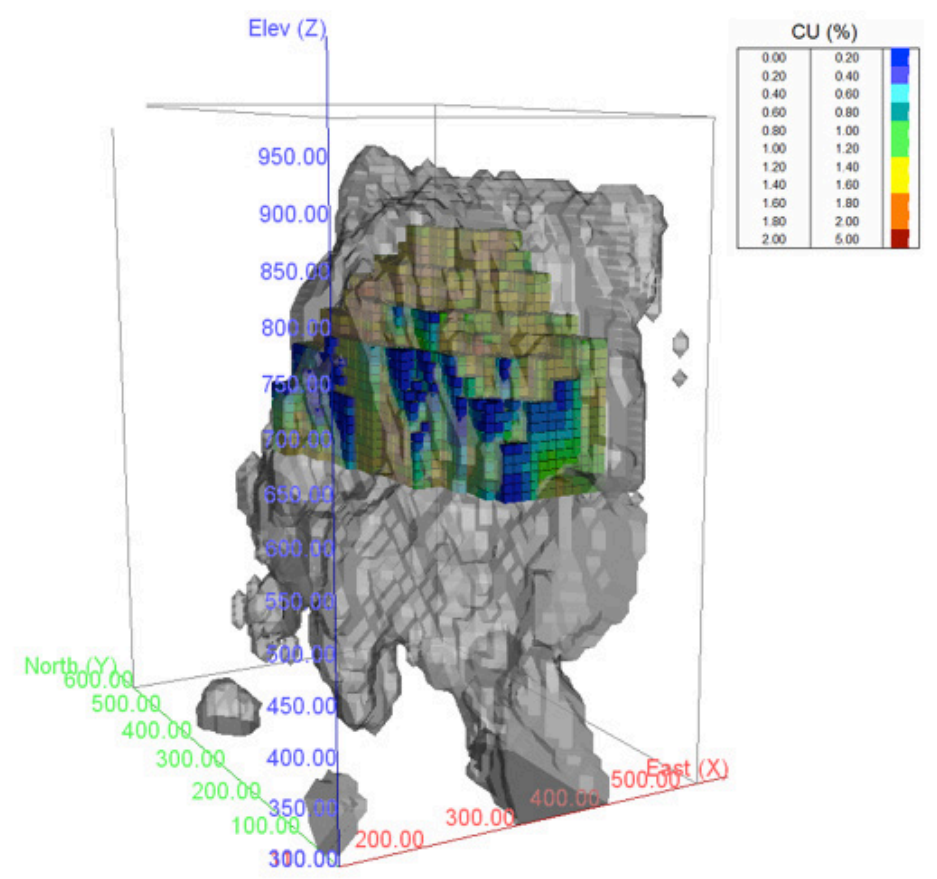

Figure 6 Caving reserves envelope (single lift) generated under geological and material flow uncertainty

The undercut opening sequence is presented in Figure 7. The total footprint area for the stochastic case with geology and flow uncertainty is $109,200 \mathrm{~m}^{2}$.

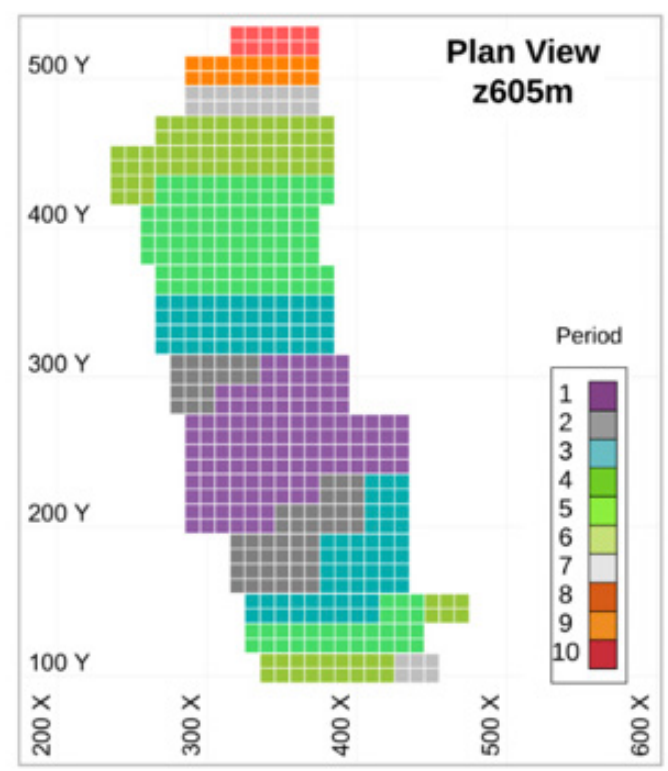

Figure 7 Undercut sequence for the stochastic case with geological and material flow uncertainty

The production schedule for the stochastic case with geological and flow uncertainty is presented in Figure 8. The ore tonnage production, at a total $26,408 \mathrm{Mt}$ meets the production targets established including the ramp-up and ramp-down periods. The average grade is shown along with the defined bounds. The overall average production grade decreases throughout the mine life, as the mining advancement direction approaches the ore/waste boundaries within the different realizations, diluting ore reserves. 


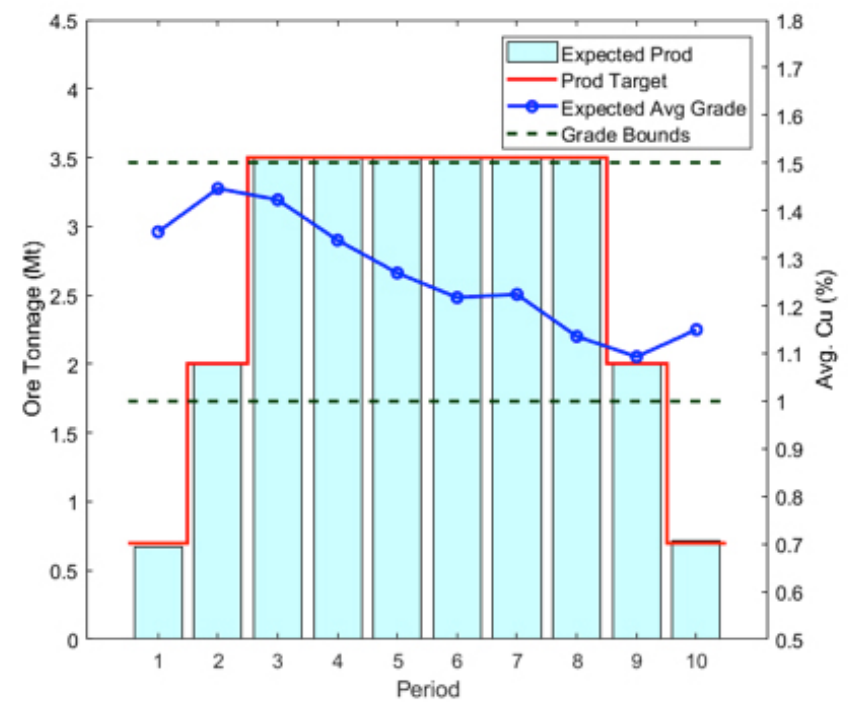

\section{Figure 8 Production schedule for the SIP model}

The cumulative NPV for the LOM plan is shown Figure 9. The expected NPV for the project under geological and flow uncertainty was found at 615,908 $\mathrm{M} \$$ with the 10th percentile of its distribution at $577,813 \mathrm{M} \$$ and the 90 th percentile at 629,316 M\$.

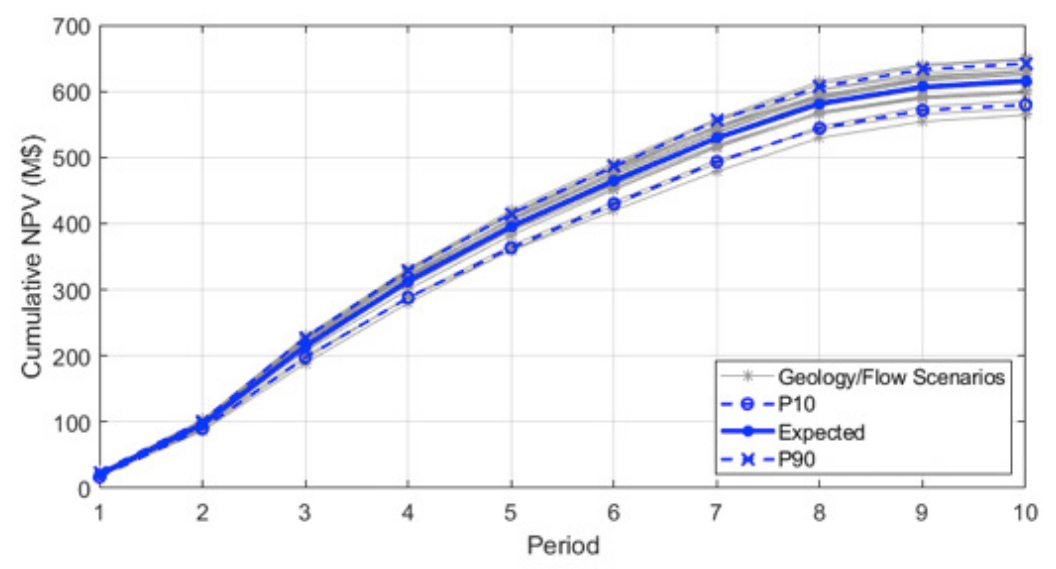

Figure 9 Cumulative NPV throughout the LOM for the multiple scenarios

The average production grade throughout the LOM for the multiple geological and flow scenarios is presented in Figure 10. All realizations were kept within the boundaries throughout the whole LOM as they were explicitly included in the optimization process, reducing the operational risk at the processing stage.

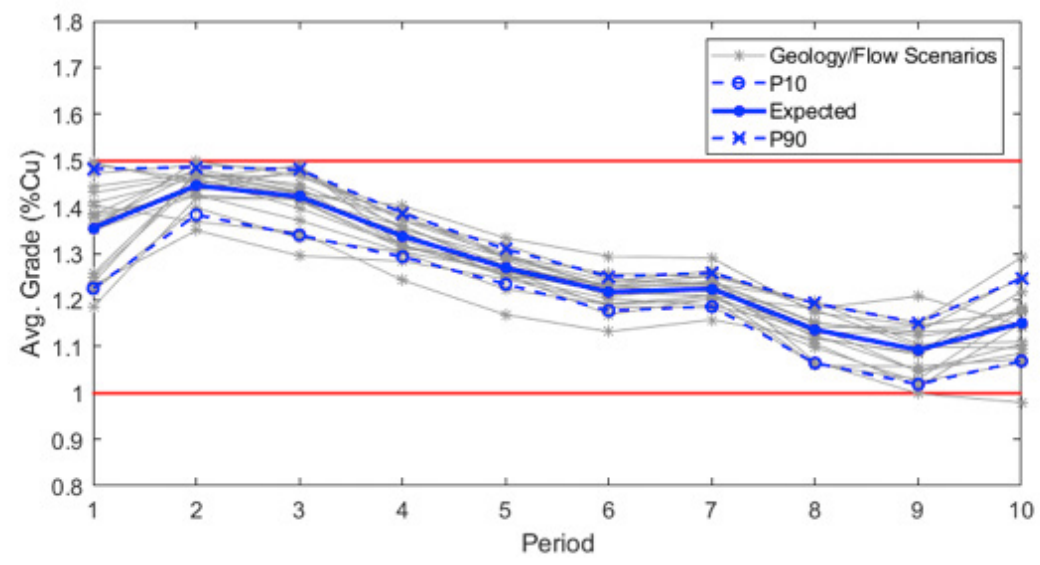

Figure 10 Average production grade throughout the LOM for the multiple scenarios 


\section{$4 \quad$ Conclusions}

A mathematical programming framework was developed to generate a LOM schedule for block caving mines from a block model scale that incorporates geological and material flow uncertainty explicitly through the use of different simulations that represent the level of information available, and the potential uncertainty due to the flow of the broken rock. The proposed workflow yields a mine plan schedule that maximizes the expected value of the project while minimizing the impact of the geological and material flow uncertainty in meeting production targets throughout the life of the mine.

\section{$5 \quad$ References}

Alvial, J 1992, 'Analysis of the extraction at El Teniente 4 Sur ', in ed. H.W. Glen, SAIMM, Proceedings of MassMin 1992, Johannesburg.

Cullenbine, C, Wood, K \& Newman, A 2011, 'A sliding time window heuristic for open-pit mine block sequencing', Optimization Letters, vol. 5, pp. 365-377.

Dimitrakopoulos, R 2011, 'Stochastic optimization for strategic mine planning: A decade of developments', Journal of Mining Science, vol. 47, no. 2, pp. 138-150.

Dirkx, R, Kazakidis, V \& Dimitrakopoulos, R 2018, 'Stochastic optimisation of long-term block cave scheduling with hang-up and grade uncertainty, International Journal of Mining, Reclamation and Environment, vol. 33, no. 6, pp. 371-388.

Khodayari, F \& Pourrahimian, Y 2019, 'Long-term production scheduling optimization and 3D material mixing analysis for block caving mines', Mining Technology, vol. 128, no. 2, pp. 65-76.

Laubscher, D, Guest, A, Jakubec, J \& Chitombo, G 2017, 'Guidelines on caving mining methods - The underlying concepts'. Brisbane, Australia.

Laubscher, D 2000, 'A practical manual on block caving', Brisbane, Australia.

Nezhadshahmohammad, F \& Pourrahimian, Y 2018, 'A clustering algorithm for block-cave production scheduling', Global Journal of Earth Science and Engineering, vol. 5, pp. 5-43.

Nezhadshahmohammad F, Pourrahimian, Y \& Aghababaei, H 2018, 'Presentation and application of a multi-index clustering technique for the mathematical programming of block-cave production scheduling', International Journal of Mining Science and Technology, vol. 28, pp. 941-950.

Osanloo, M, Gholamnejad, J \& Karimi, B 2008, 'Long-term open pit mine production planning: A review of models and algorithms', International Journal of Mining, Reclamation and Environment, vol 22, no. 1, pp. 3-35.

Pourrahimian, Y, Askari-Nasab, H \& Tannant, D 2013, 'A multi-step approach for block-cave production scheduling optimization', International Journal of Mining Science and Technology', vol.23, no. 5, pp. 739-150.

Tabesh, M \& Askari-Nasab H 2011, 'Two-stage clustering algorithm for block aggregation in open pit mines', Transactions of the Institution of Mining and Metallurgy, Section A: Mining Technology, vol. 120, no. 3, pp. 158-169.

Topal, E 2008, 'Early start and late start algorithms to improve the solution time for long-term underground mine production scheduling', The journal of The Southern African Institute of Mining and Metallurgy, vol. 108, pp. 99-107.

Weintraub, A, Pereira, M \& Schultz, X 2008, 'A priori and a posteriori aggregation procedures to reduce model size in MIP Mine planning models', Electronic Notes in Discrete Mathematics, vol. 30, pp. 297-302. 